\title{
Anemia in End Stage Renal Disease
}

\author{
Mojgan Jalalzadeh ${ }^{1, *}$ \\ ${ }^{1}$ Metropolitan Hospital Center, New York Medical College, New York, United States \\ "Corresponding author: Metropolitan Hospital Center, New York Medical College, New York, United States. Email: jmojgan1341@gmail.com
}

Received 2020 September 16; Accepted 2020 November 15.

Keywords: Anemia, Chronic Kidney Disease (CKD), End Stage Renal Disease (ESRD)

\section{Dear Editor,}

Anemia is a common complication of chronic kidney disease (CKD) and can appear before uremic syndrome when the glomerular filtration rate (GFR) decreases to less than $30 \mathrm{~mL} / \mathrm{min}$.

A low level of hemoglobin $(\mathrm{Hb})$ is an independent risk factor for low quality of life in CKD patients. Untreated anemia in CKD patients leads to several physiological abnormalities such as impaired heart function, decreased cognation and mental activity, hemostatic dysfunction, impaired immune function and sexual function.

Clinical symptoms of anemia include fatigue, depression, concentration problems, loss of appetite, sleep disturbance, shortness of breath and heart palpitations.

Anemia is associated with higher rate of cardiovascular mortality in patients with CKD. Cardiovascular mortality is due to left ventricular hypertrophy (LVH). Every $1 \mathrm{~g} / \mathrm{dL}$ reduction in $\mathrm{Hb}$ concentration is associated with 6 percent increase in risk of LVH (1), particularly when the serum $\mathrm{Hb}$ level is $<10 \mathrm{~g} / \mathrm{dL}$.

The guidelines of kidney disease outcome quality initiative (K/DOQI) of National Kidney foundation (NKF)) recommend that $\mathrm{CKD}$ cases with $\mathrm{Hb}<13.5 \mathrm{~g} / \mathrm{dL}$ in men and $<12 \mathrm{~g} / \mathrm{dL}$ in women need to have anemia assessment (2). The risk of morbidity and mortality decrease in CKD cases at $\mathrm{Hb}$ level > $11 \mathrm{~g} / \mathrm{dL}$. Studies have shown that anemia correction has no effect on progression from kidney failure to end stage renal disease (ESRD). In correcting anemia, if $\mathrm{Hb}$ $>13 \mathrm{~g} / \mathrm{dL}$ happens, then the risk of adverse outcome such as cardiovascular events, stroke and death will be increased.

The main reason for anemia among CKD patients is erythropoietin (EPO) insufficiency, which is due to insufficient production of EPO by the endothelial cells in proximal tubules. Other causes of anemia in ESRD patients are iron deficiency and inflammation. The current approach to correcting anemia is using minimum value of erythropoeisisstimulating agents (ESAs) along with the treatment of iron deficiency and inflammation. The main goal of ESAs therapy is to reduce the need for blood transfusion, which has side effects such as transfusion reaction, viral infection, iron overload and immune sanitization.

The KDIGO guidelines recommend keeping $\mathrm{Hb}$ levels between 9 and $11.5 \mathrm{~g} / \mathrm{dL}$ and when $\mathrm{Hb}<10 \mathrm{~g} / \mathrm{dL}$, ESAs therapy be started. ESAs therapy provides clinical benefits such as increased energy levels, improved quality of life, increased exercise ability, work and sexual activity, reduced depression, and fatigue. However, in patients with CKD who have a history of stroke or cancer, the use of ESAs therapy should be with caution.

There are short-acting and long-acting ESAs therapies. The short-acting ESA is called Epoetin alfa (recombinant human EPO, rHUEpo), which has a half-life of 8 hours when given intravenously and 16 - 41 hours when given subcutaneously. For hemodialysis (HD) patients, rHuEpo should be started with a dosage of 2000 - 3000 units subcutaneously three times per week. For a peritoneal dialysis (PD) patients rHuEpo should be started with a dosage of 6000 units subcutaneously once per week, which is the main approach. The subcutaneous administration of short acting ESAs reduces the required dose of ESAs by $25 \%$, whereas, IV injection reduces patients' discomfort.

The Darbepoeitin alfa is the long-acting ESAs, which has a longer half-life than rHuEPO agents. Its half-life is about 25 hours when given through IV and50 hours when given subcutaneous. The started dosage in CKD patients is 20 - 30 mcg per week or 40 - 60 mcg every two weeks. The darbepoeitin alfa half-life is threefold longer than rHuEPO agents and has greater biological activity $(3,4)$. 
Patients' Hb should be monitored every 1 - 2 weeks and ESA dosage should be adjusted as needed. After first month of ESAs therapy, if Hb level is not increased, it would represent hypo-responsiveness to ESA. For this situation, KDIGO recommends that the dosage of ESA should not be increased more than twice the initial value used and other causes for anemia should be considered.

Iron deficiency anemia is the most common cause of hypo responsiveness toESA in CKD patients, who have iron deficiency. Iron deficiency is due to several factors such as reduced iron absorption, blood loss due to repeated blood draws and gastrointestinal blood loss, and reduced nutritional consumption due to loss of appetite. There are two types of iron deficiency anemia called absolute iron deficiency and functional iron deficiency.

Absolute iron deficiency represents lack of iron in the bone marrow. It can be diagnosed by bone marrow biopsy or by using transferring saturation (TSAT) $\leq 20 \%$ (serum iron $\times 100 /$ total iron binding capacity) and serum ferritin concentration $\leq 100 \mathrm{ng} / \mathrm{mL}$ in PD patients and $\leq 200$ $\mathrm{ng} / \mathrm{mL}$ among HD patients.

Functional iron deficiency indicates insufficient absorption of iron by erythroid precursors when adequate iron exists in bone marrow. It consists of two types. The first type depends to ESA management, which may respond to IV iron therapy; and second type is related to anemia of chronic disease, which does not improve by iron administration. Both types of functional iron deficiency are characterized by TSAT $\leq 20 \%$ and serum ferritin concentration $\geq 800 \mathrm{ng} / \mathrm{mL}$.

Treatment iron deficiency anemia. Oral iron therapy is the desired method for non dialysis CKD patients. It is recommended that patients take $200 \mathrm{mg}$ of iron element per day, which is equivalent to $325 \mathrm{mg}$ ferrous sulfate three times per day on an empty stomach. For improving absorption of iron, it should not be taken with phosphate binders. The oral iron supplement should not be used in HD cases because of poor efficacy, constipation, dyspepsia, bloating and diarrhea.

The appropriate intravenous iron supplements are low molecular weight dextran, ferrous glocunate, iron sucrose, or feroumoxytol. The use of high molecular weight iron dextran increases the risk of anaphylaxis. The first course of IV iron supplement is $1000 \mathrm{mg}$ of iron over 8-10 consecutive HD sessions, which can be repeated if the initial course of treatment fails to increase Hb level. Then, IV iron with a dosage of 25-100 mg should be administered per week.

Patients who require high doses of ESAs are called ESA resistant patients. High dosage of ESAs therapy increases the risk of side effects such as worsening of hypertension, seizure, stroke, graft clotting, change of Kt/V and high levels of serum inflammatory markers, which is associated with poor prognosis of survival.

Causes of ESA resistance include bleeding, short lifespam of RBC in dialysis patients, infection and inflammation, vitamin D deficiency, vitamin B12 and folic acid deficiency, aluminum intoxication, consumption of ACE inhibitors, pure red cell aplasia (it is associated with Epoetin alfa), and other hematologic causes (malignancy, myelodysplastic syndrome, hemolysis).

Intravenous administration of ascorbic acid may improve response to ESAs therapy and reduce the dosage of ESA. It should be noted that the administration of vitamin $C$ supplements result in production of oxalate and its usage should be with caution during treatment.

\section{Footnotes}

Authors' Contribution: Mojgan Jalalzadeh was the only author of this manuscript.

Conflict of Interests: None.

Funding/Support: This study was supported by a teaching and research from the Metropolitan Hospital Center, New York Medical College, New York, NY, United States.

\section{References}

1. Levin A, Singer J, Thompson CR, Ross H, Lewis M. Prevalent left ventricular hypertrophy in the predialysis population: identifying opportunities for intervention. Am J Kidney Dis. 1996;27(3):347-54. doi: 10.1016/s0272-6386(96)90357-1. [PubMed: 8604703].

2. Go AS, Chertow GM, Fan D, McCulloch CE, Hsu CY. Chronic kidney disease and the risks of death, cardiovascular events, and hospitalization. N Engl J Med. 2004;351(13):1296-305. doi: 10.1056/NEJMoa041031. [PubMed: 15385656].

3. Locatelli F, Olivares J, Walker R, Wilkie M, Jenkins B, Dewey C, et al. Novel erythropoiesis stimulating protein for treatment of anemia in chronic renal insufficiency. Kidney Int. 2001;60(2):741-7. doi: 10.1046/j.1523-1755.2001.060002741.x. [PubMed: 11473657].

4. Carrera F, Burnier M. Use of darbepoetin alfa in the treatment of anaemia of chronic kidney disease: clinical and pharmacoeconomic considerations. NDT Plus. 2009;2(Suppl_1):i9-i17. doi: 10.1093/ndtplus/sfn175. [PubMed: 19461859]. [PubMed Central: PMC2638549]. 